\title{
The $1430 \mathrm{~cm}^{-1}$ Raman line in single crystals of $\mathrm{La}_{2} \mathrm{CuO}_{4-y}$
}

I. Ohana and M.S. Dresselhaus ${ }^{\text {a) }}$

Department of Physics, Massachusetts Institute of Technology, Cambridge, Massachusetts 02139

D. Heiman and G. Dresselhaus

Francis Bitter National Magnet Laboratory, Massachusetts Institute of Technology, Cambridge, Massachusetts 02139

P. J. Picone ${ }^{\text {b) }}$

Center for Materials Science and Engineering, Massachusetts Institute of Technology, Cambridge, Massachusetts 02139

(Received 14 November 1988; accepted 23 January 1989)

The Raman line at $1430 \mathrm{~cm}^{-1}$ (M-line) in single crystal $\mathrm{La}_{2} \mathrm{CuO}_{4-y}$ was studied as a function of doping, temperature, magnetic field, and excitation wavelength. Upon Li doping the line becomes broader, and it vanishes rapidly with Sr-doping. The line also broadens with increasing temperature and increasing applied magnetic field. Resonance enhancement was found for decreasing laser excitation energies but was not as pronounced as the enhancement of several alleged two-phonon lines. Many of these features are correlated with the 2D antiferromagnetic ordering measured in this system by neutron scattering. The possible identification of this line as a one-spin excitation is favored by the data though a two-phonon excitation is also considered.

\section{INTRODUCTION}

Light scattering provides information on different types of elementary excitations in solids such as lattice, magnetic, and electronic excitations. $\mathrm{La}_{2} \mathrm{CuO}_{4-y}$ shows a very strong magnetic interaction, ${ }^{1}$ unusual electronic properties, and a strong electron-lattice interaction. Therefore, any peaks in the Raman spectra of $\mathrm{La}_{2} \mathrm{CuO}_{4-y}$ must be examined carefully in order to identify the origin of the excitation. In this paper, we focus only on the $1430 \mathrm{~cm}^{-1}$ line (M-line) which is the strongest feature in the Raman spectrum of $\mathrm{La}_{2} \mathrm{CuO}_{4-y}$ in the range of $\omega<2000 \mathrm{~cm}^{-1}$ taken with the $(x x)$ polarization configuration. ${ }^{2-4}$ (See Fig. 1.) We report here several properties of this line: the dependence of the line on $\mathrm{Li}$ and $\mathrm{Sr}$ doping; the dependence on temperature; a small effect of a magnetic field on the lineshape of the line; and we also compare the resonance enhancement, as the laser excitation energy is varied, to that of other lines. The possible identification of this line as either a one-spin magnetic excitation or a two-phonon excitation is discussed.

\section{EXPERIMENTAL DETAILS}

The measurements presented here were performed on single crystals which were grown from flux by the top seeded solution technique described elsewhere. ${ }^{5}$ All the crystals were characterized using $x$-ray diffraction and Laue diffraction patterns. Although the Laue patterns show clear Bragg spots, twinning and oxygen vacancies were

\footnotetext{
a) Also Department of Electrical Engineering and Computer Science.

b) Permanent address: Defense Science Technology Organization, Adelaide, Australia.
}

also likely present. The crystals were further characterized by magnetic susceptibility measurements. A small cusp associated with the 3D magnetic ordering was observed in the undoped crystal at $237 \mathrm{~K}$. However, the magnetic susceptibility measurement on the Li-doped sample showed a strong suppression of this cusp, indicating that the $3 D$ magnetic ordering is almost completely suppressed upon Li doping. ${ }^{6}$ The undoped and Li-doped single crystals show similar temperature-dependent resistivities and exhibit insulator-like behavior. ${ }^{4,7}$ The two single crystals, containing $1.5 \%$ and $3 \% \mathrm{Sr}$, were not superconducting; however, they are much more metallic than the undoped sample. The resistivity $\rho$ of the $3 \% \mathrm{Sr}$-doped sample is almost temperature independent and at $10 \mathrm{~K}$, the resistivity $\rho$ is smaller by four orders of magnitude compared with typical resistivity values for the undoped crystals at the same temperature.

All the Raman spectra of the doped and undoped single crystals were taken in a backscattering configuration with the 4880 and the $5145 \AA$ lines of an $\mathrm{Ar}^{+}$ion laser. The high-quality undoped crystal was also studied with different laser excitation energies obtained from $\mathrm{Ar}^{+}, \mathrm{Kr}^{+}$, and dye lasers. The laser beam was focused with a lens of $10 \mathrm{~cm}$ focal length to a spot size smaller than 100 microns. The laser was kept at a power level below $35 \mathrm{mw}$ to avoid local heating. The spectra were detected by a $0.85 \mathrm{~m}$ double monochromator, equipped with a single channel photon counting system.

\section{RESULTS AND DISCUSSION}

In Fig. 1 we present polarized Raman spectra of undoped, $4 \% \mathrm{Li}$-doped, and $3 \% \mathrm{Sr}$-doped $\mathrm{La}_{2} \mathrm{CuO}_{4-y}$ samples, 


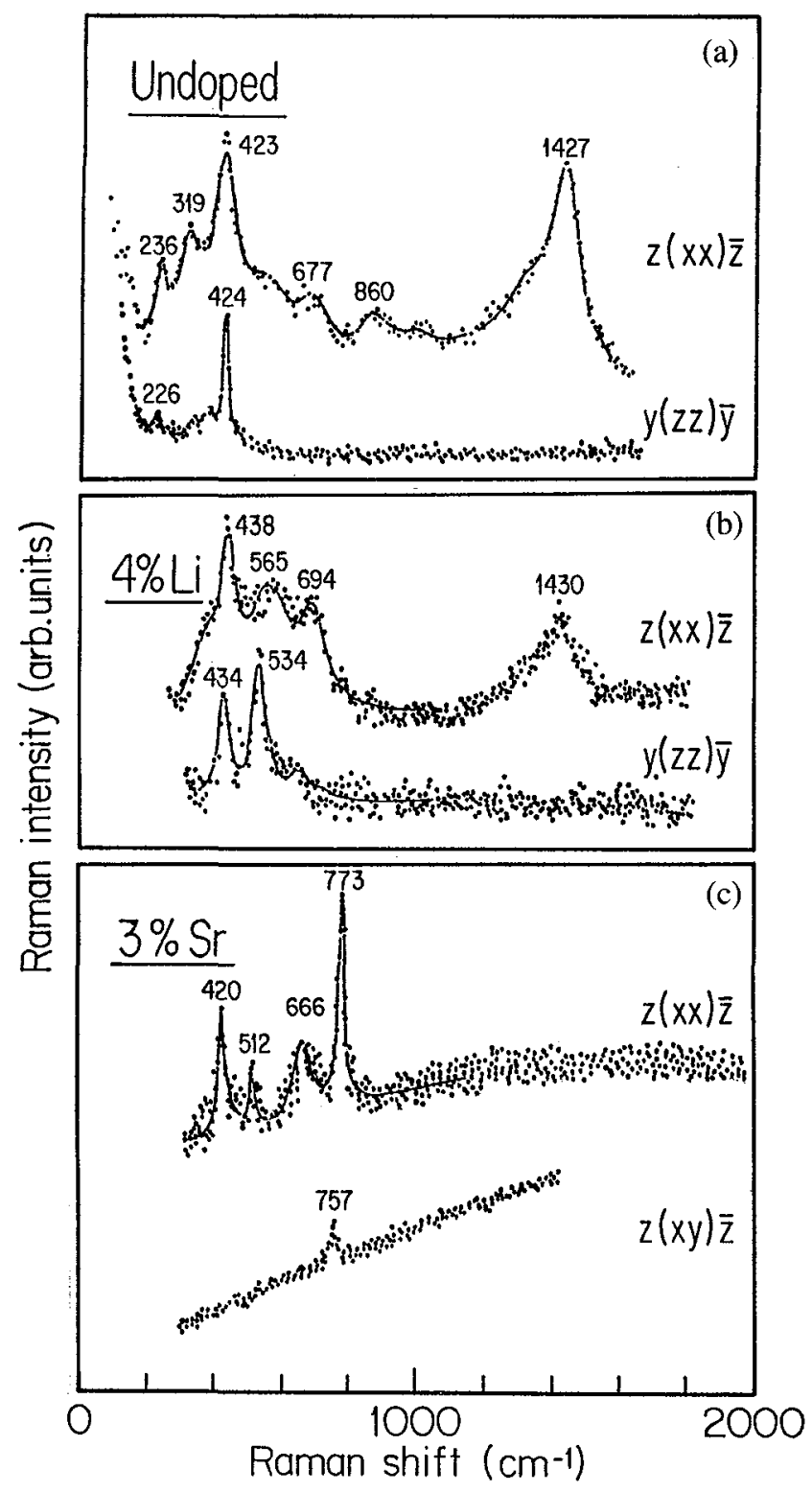

FIG. 1. Polarized Raman spectra of (a) undoped, (b) Li-doped, and (c) Srdoped single crystals of $\mathrm{La}_{2} \mathrm{CuO}_{4-y}$. All the spectra were taken at room temperature with the $4880 \AA$ line of an $\mathrm{Ar}^{+}$ion laser. Our main focus is the $\mathrm{M}$-line at $1430 \mathrm{~cm}^{-1}$

where $y$ denotes small deviations from oxygen stoichiometry and can be either positive or negative. The spectra presented in Fig. 1 were taken in the $z(x x) \bar{z}$ and $y(z z) \bar{y}$ configurations for the first two samples and in the $z(x x) \bar{z}$ and $z(x y) \bar{z}$ configurations for the Sr-doped crystal. The $z$ direction is the tetragonal $\hat{c}$ axis while $x$ and $y$ refer to the inplane axes along the $\mathrm{Cu}-\mathrm{O}$ bonds. The notation $z(x y) \bar{z}$, for example, describes the scattering configuration where the incident beam is along the $z$ direction with the electric field vector $\mathbf{E}$ along the $x$ axis, and the scattered beam is along the $\bar{z}$ direction with $\mathbf{E}$ parallel to $y$. All the spectra in this figure were measured with a laser excitation wavelength of $4880 \AA$. The lines in the low-frequency region of the spec- tra are associated with phonons and were discussed elsewhere. ${ }^{4}$ Here we focus our attention on the Raman line at $1430 \mathrm{~cm}^{-1}$ (the M-line). From these spectra and polarized measurements in the $\left(x^{\prime} x^{\prime}\right)$ and $\left(x^{\prime} y^{\prime}\right)$ configurations (where $x^{\prime}, y^{\prime}$ denote the axes rotated by $45^{\circ}$ with respect to $x, y$ ) we conclude that the line must have $A_{1}$ symmetry.

The characteristics of the M-line depend strongly on doping. The line is broadened in the Li-doped samples and is not observed in either the $1.5 \%$ or the $3 \%$ Sr-doped samples. In Fig. 2 we see that the room temperature linewidth increases by a factor of three as the $\mathrm{Li}$ doping increases to about 6\%. From Fig. 1 we also notice that the intensity of the M-line relative to the intensity of the firstorder Raman-active phonon $\left(\sim 425 \mathrm{~cm}^{-1}\right)$ decreases upon $\mathrm{Li}$ doping. In a recent paper, it was shown that the M-line depends also on the oxygen content, ${ }^{8}$ with the line vanishing in a Li-doped sample $(\sim 3 \%)$ which was annealed in oxygen at $1000^{\circ} \mathrm{C}$ for $24 \mathrm{~h}$.

We further measured the variation of the line position and width as a function of temperature. A large effect (see Fig. 3) was found in the sample containing about $6 \% \mathrm{Li}$.

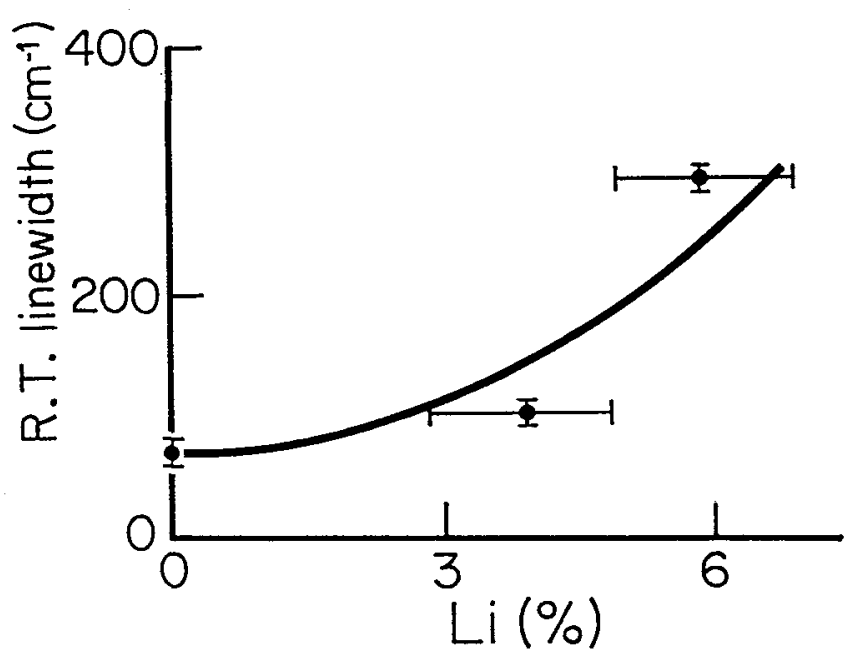

FIG. 2. The dependence of the room temperature linewidth (full-width at half-maximum intensity) of the Raman line at $1430 \mathrm{~cm}^{-1}$ upon Li doping.

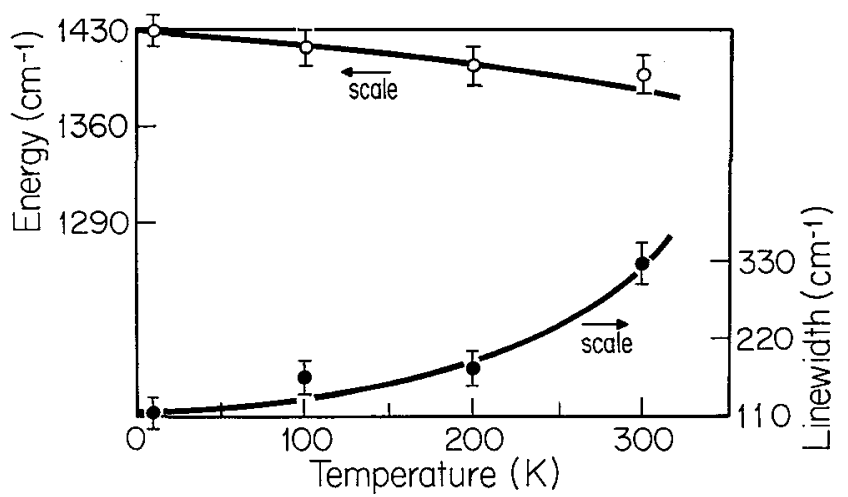

FIG. 3. The temperature variation of the energy (frequency) and the linewidth (full-width at half-maximum intensity) of the $1430 \mathrm{~cm}^{-1}$ line in the $6 \% \mathrm{Li}$-doped single crystal of $\mathrm{La}_{2} \mathrm{CuO}_{4-y}$. 
Although the frequency of the line slightly decreases with increasing temperature, the linewidth dramatically increases, showing an increase by almost a factor of three when the temperature is varied from $10 \mathrm{~K}$ to $300 \mathrm{~K}$. In contrast, the lineshape of the undoped sample exhibits only a small variation with temperature, namely a monotonic increase in the linewidth from about 50 to $60 \mathrm{~cm}^{-1}$ fullwidth at half-maximum (FWHM) upon increasing the temperature from $4 \mathrm{~K}$ to room temperature.

Motivated by the observed magnetism in this material (e.g., Endoh et al. ${ }^{6}$ and references cited therein), we looked for magnetic effects on the lineshape of all the lines in the Raman spectra. Since we were limited to magnetic fields of 10 tesla, we might not expect a strong effect since the exchange interaction, $J$, is too high $\left(\sim 700-1000 \mathrm{~cm}^{-1}\right) .^{2,9}$ We, however, observed a small change in the lineshape of the $\mathbf{M}$-line in a magnetic field $(\mathbf{H} \perp \hat{c})$. No effect was found for the one-phonon line at $\sim 425 \mathrm{~cm}^{-1}$, the twophonon line at $\sim 1200 \mathrm{~cm}^{-1}$, and for the configuration $\mathbf{H} \| \hat{c}$. In Fig. 4 we see that the intensity of the line decreases and the linewidth increases when the magnetic field increases from 6 tesla to 10 tesla; there is no detectable change in linewidth or intensity from $H=0$ to $H=6 \mathrm{~T}$. The variation of the linewidth and the intensity of the M-line, calibrated with respect to the $1200 \mathrm{~cm}^{-1}$ line, for various magnetic fields is plotted in the inset of Fig. 4.

Remarkable differences are found between the Raman spectra, taken at different laser excitation energies $\omega_{L}$, as shown by the two spectra in Fig. 5. The upper spectrum was taken with $\lambda_{L}=5145 \AA$ and the lower one with

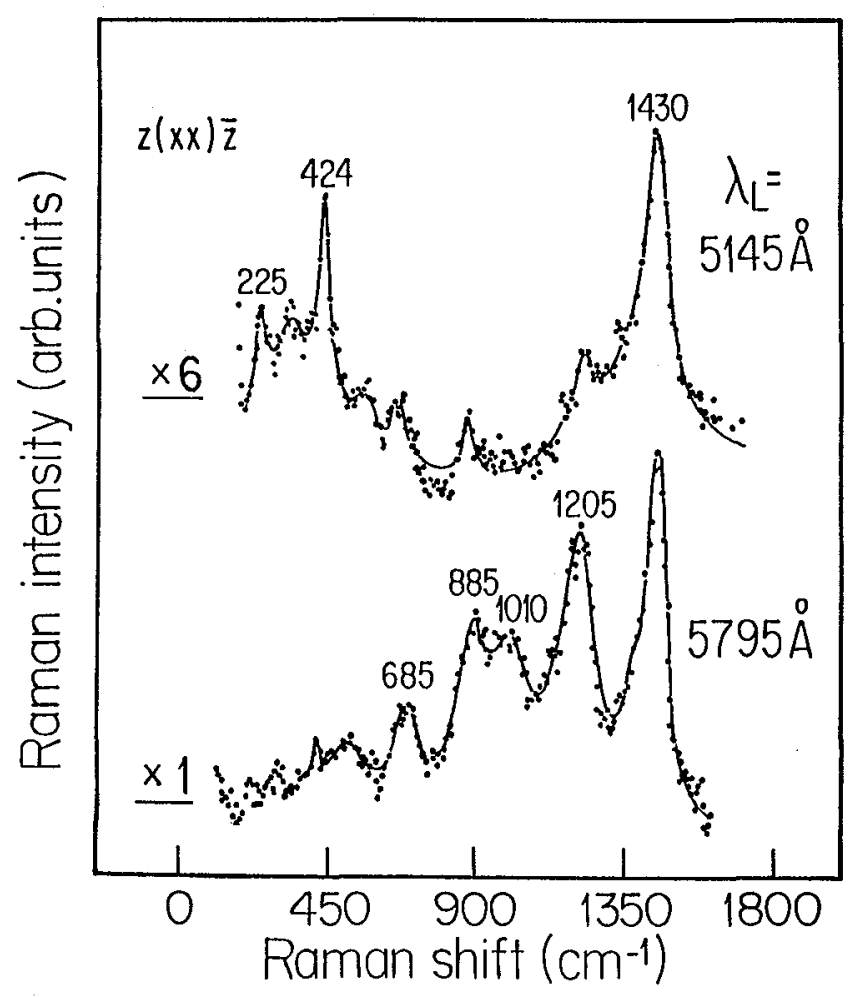

FIG. 5. Polarized room temperature Raman spectra of single crystal $\mathrm{La}_{2} \mathrm{CuO}_{4-y}$. The upper spectrum was taken with a $5145 \AA$ laser line and the lower with a $5795 \AA$ laser line. Note the increased scale factor of the upper spectrum.

$\lambda_{L}=5795 \AA$. All the lines that exhibit an intensity enhancement relative to the M-line are associated with a

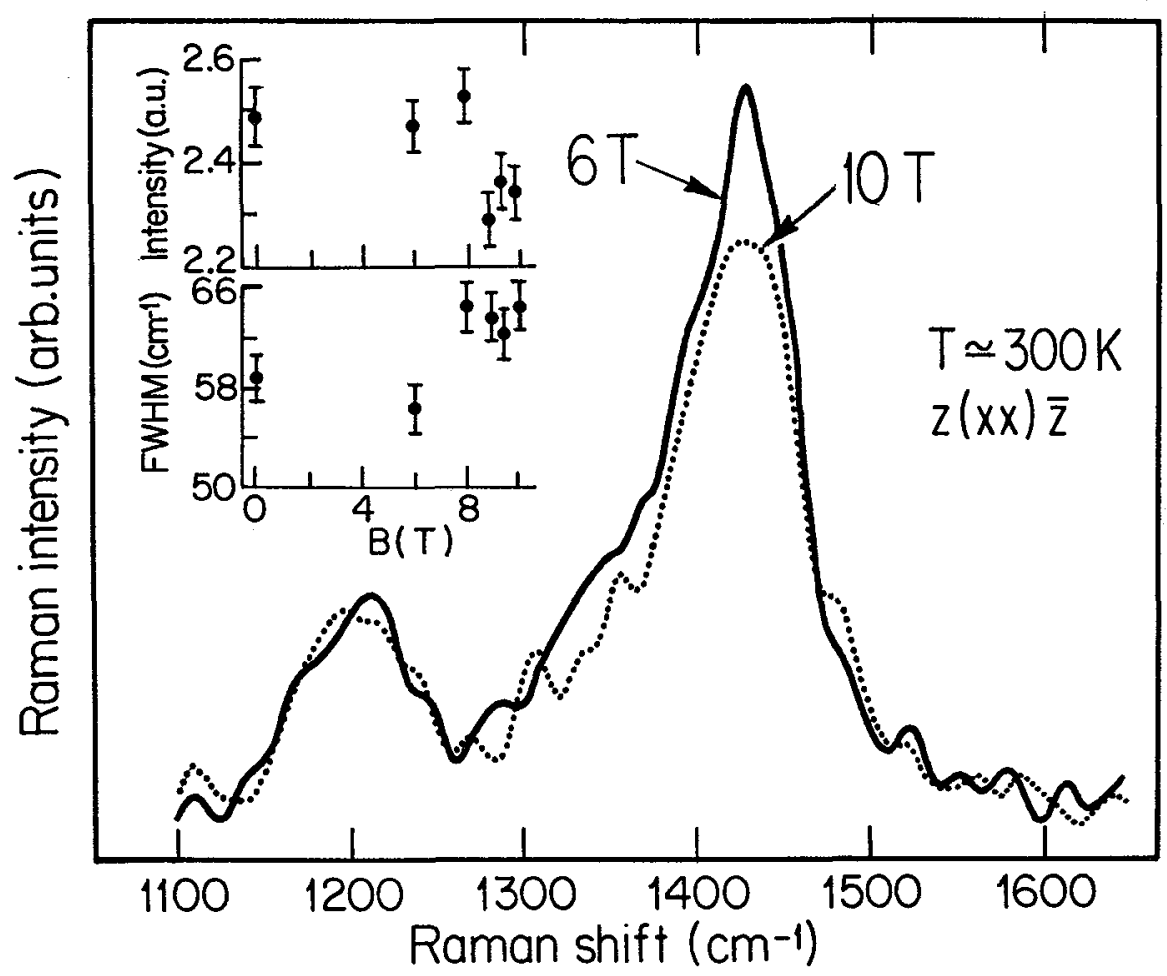

FIG. 4. The change in the lineshape of the $1430 \mathrm{~cm}^{-1}$ Raman line under an applied magnetic field $(\mathbf{H} \perp \hat{c})$. The spectra were taken at room temperature with the $4880 \AA$ $\mathrm{Ar}^{+}$ion laser line and with the $z(y y) \bar{z}$ scattering configuration. In the inset the variation of the intensity and linewidth is given as a function of magnetic field. 
resonant effect due to an electronic gap at $2.1 \mathrm{eV}{ }^{10}$ In the present study, we emphasize the difference in behavior between the M-line and the other high-frequency lines. Figure 6 shows the integrated intensities of the 883 and $1205 \mathrm{~cm}^{-1}$ lines relative to that of the M-line.

The properties of the $\mathbf{M}$-line can be summarized as follows:

(1) $A_{1}$ symmetry;

(2) Linewidth broadened upon Li doping;

(3) Not observed for samples containing $1.5 \%$ and $3 \% \mathrm{Sr}$, and for sample heavily annealed in oxygen;

(4) Broader linewidths for increasing temperature (especially pronounced for $\mathrm{Li}$ doping);

(5) Small variation of the lineshape with application of a magnetic field;

(6) Different dependence of the line intensity on laser excitation energy relative to other Raman lines.

On the basis of the above properties we suggest that the $\mathrm{M}$-line has a magnetic origin. The identification of the line as a first-order phonon excitation seems unlikely because its energy is too high; its energy scale, however, is consistent with a possible multi-phonon excitation. We have two experimental arguments against the multi-phonon explanation: first, the M-line shows a magnetic field-dependent effect (though small). In principle, one can argue that the magnetic field effect on the lineshape of the M-line may originate from magnetostriction, namely a change in the lattice constant associated with a magnetic phase transi-

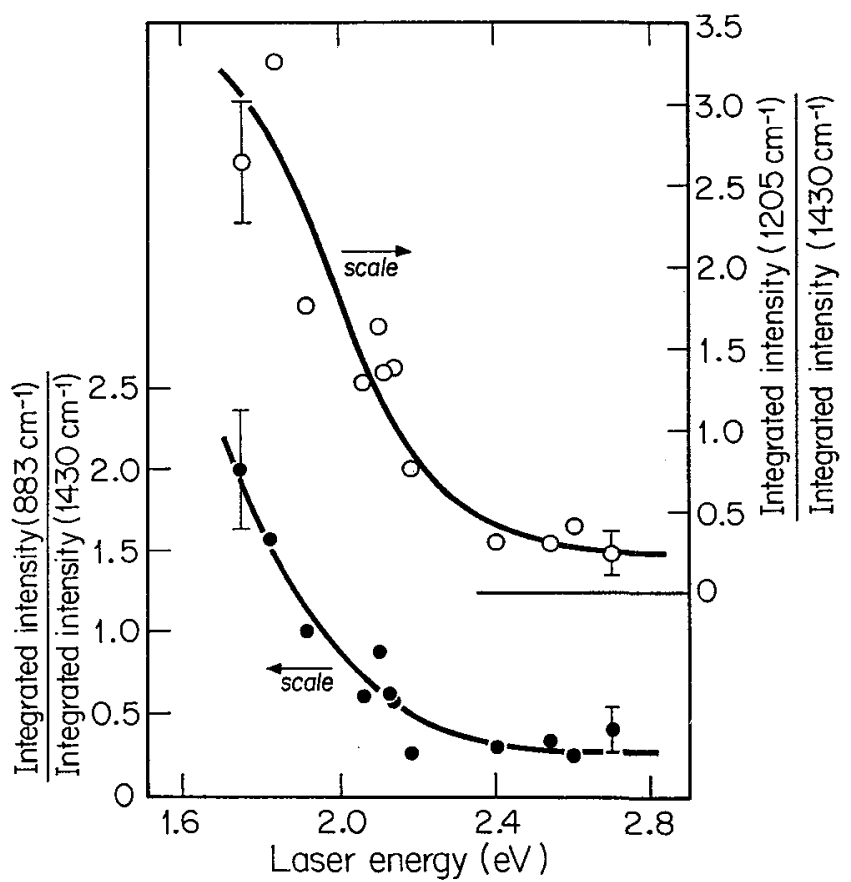

FIG. 6. Ratio of the integrated intensities of the (a) $883 \mathrm{~cm}^{-1}$ and (b) $1205 \mathrm{~cm}^{-1}$ Raman lines relative to the $1430 \mathrm{~cm}^{-1}$ line as a function of laser excitation energy. tion. However, it was recently reported that the magnetostriction effect in $\mathrm{La}_{2} \mathrm{CuO}_{4-y}$ is very small: $\Delta a / a$ is less than $10^{-7} .^{11}$ Such changes in the lattice constants are too small, by at least three orders of magnitude, to be seen in the Raman spectra. The second argument against the multiphonon explanation is related to the very different dependence of the M-line intensity on laser excitation energy compared with other Raman lines, as is shown in Fig. 6. However, since the electronic bands of this material are yet not known, there remains the possibility that different Raman lines can show different enhancements at other electronic gaps.

The suggestion of the magnetic origin of the M-line is based on the correlation between the properties of this line and the properties of the 2D magnetic correlation phenomena reported by the MIT/NTT/Brookhaven group, ${ }^{1,6,9}$ who found that the 2D magnetic correlation length strongly depends on doping. In the undoped material, they found the correlation length, $\xi$, to be $\approx 200 \AA$. Upon Li doping $\xi$ decreases to about $50 \AA$, and in the Sr-doped crystal, they found $\xi$ to be very small $(\xi \leqslant 20 \AA)$.

The properties of the M-line are consistent with the behavior of the $2 \mathrm{D}$ antiferromagnetic correlation for the following reasons: the line becomes broader upon Li doping, consistent with the shorter $\xi$, and completely disappears in the Sr-doped crystals which have yet shorter $\xi$. From symmetry considerations we can exclude the possibility that the line is a two-spin excitation, since such excitations are not expected on the basis of Parkinson's theory ${ }^{12}$ to possess $A_{1}$ symmetry. The line may, however, be due to a magnetic defect-induced one-spin excitation. This is an excitation of a spin which is confined to a small area in real space by some defect in the magnetic ordering. For such a defect the excitation energy would be $S J$ times the number of interacting nearest neighbors, which is three since one of the nearest neighbors has a defect. We thus obtain for the energy $E_{M}$ of the M-line $\sim 3 S J$, and by taking $S=1 / 2$ we get $J=950 \mathrm{~cm}^{-1}$ for the nearest neighbor intraplanar antiferromagnetic interaction which is consistent with the various suggested values for $J$ from the literature, ${ }^{2,9} \sim 750<J<1100 \mathrm{~cm}^{-1}$.

In conclusion, various properties of the Raman line at $1430 \mathrm{~cm}^{-1}$ have been examined in terms of two-phonon and magnetic origins. From the present results, we cannot completely eliminate the two-phonon mechanism; however, the current evidence favors the identification of the $\mathrm{M}$-line as a defect-induced one-spin excitation.

\section{ACKNOWLEDGMENTS}

The authors are grateful for fruitful discussions with Dr. H. Zeiger. The work at Massachusetts Institute of Technology has been supported by NSF grant DMR87-19217. Part of the measurements was carried out at the Francis 
Bitter National Magnet Laboratory, which is supported by the NSF grant DMR85-11789.

\section{REFERENCES}

'G. Shirane, Y. Endoh, R. J. Birgeneau, M. A. Kastner, Y. Hidaka, M. Oda, M. Suzuki, and T. Murakami, Phys. Rev. Lett. 59, 1613 (1987).

${ }^{2}$ K. B. Lyons, P. A. Fleury, J. P. Remeika, A. S. Cooper, and T. J. Negran, Phys. Rev. B 37, 2353 (1988).

${ }^{3}$ W. H. Weber, C. R. Peters, B. M. Wanklyn, C. Chen, and B. E. Watts, Phys. Rev. B 38, 917 (1988).

${ }^{4}$ I. Ohana, M. S. Dresselhaus, Y.C. Liu, P. J. Picone, D. R. Gabbe, H. P. Jenssen, and G. Dresselhaus, Phys. Rev. B 39, 2293 (1989)

${ }^{5}$ P. J. Picone, H. P. Jenssen, and D. R. Gabbe, J. Cryst. Growth 85, 576 (1987).

${ }^{6} \mathrm{Y}$. Endoh, K. Yamada, R. J. Birgeneau, D. R. Gabbe, H. P. Jenssen, M. A. Kastner, C. J. Peters, P. J. Picone, T. R. Thurston, J. M. Tran- quada, G. Shirane, Y. Hidaka, M. Oda, Y. Enomoto, M. Suzuki, and T. Murakami, Phys. Rev. B 37, 7443 (1988).

${ }^{7}$ M. A. Kastner, R. J. Birgeneau, C. Y. Chen, Y. M. Chiang, D. R. Gabbe, H. P. Jenssen, T. Junk, C. J. Peters, P. J. Picone, T. Thio, T. R. Thurston, and H. L. Tuller, Phys. Rev. B 37, 111 (1988).

${ }^{8}$ I. Ohana, Y. Liu, M. S. Dresselhaus, G. Dresselhaus, A. J. Strauss, H. J. Zeiger, P. J. Picone, H. P. Jenssen, and D. R. Gabbe, in High Temperature Superconductors, edited by M. B. Brodsky, R. C. Dynes, K. Kitazawa, and H. L. Tuller (Materials Research Society, Pittsburgh, PA, 1988), Vol. 99, p. 439.

${ }^{9}$ C. J. Peters, R. J. Birgeneau, M. A. Kastner, H. Yoshizawa, Y. Endoh, J. Tranquada, G. Shirane, Y. Hidaka, M. Oda, M. Suzuki, and T. Murakami, Phys. Rev. B 37, 9761 (1988)

${ }^{10} \mathrm{I}$. Ohana, D. Heiman, M. S. Dresselhaus, and G. Dresselhaus (1988) (unpublished research).

"N. F. Oliveira, Jr., J. T. Nicholls, Y. Shapira, G. Dresselhaus, M.S. Dresselhaus, P. J. Picone, D. R. Gabbe, and H. P. Jenssen, Phys. Rev. B 39, 2898 (1989).

${ }^{12}$ J. R. Parkinson, J. Phys. C. 2, 2012 (1969). 